\title{
Antiferromagnetic order and dielectric gap within the vortex core of antiferromagnetic superconductor
}

\author{
V. V. Garkusha and V. N. Krivoruchko* \\ Donetsk National Universty, Str. \\ Universitetskaya 24, 83055 Donetsk, Ukraine \\ * Donetsk Physics 85 Technology Institute NAS of Ukraine, \\ Str. R. Luxemburg 72, 83114 Donetsk, Ukraine
}

\begin{abstract}
The structure of a superconducting vortex has been studied theoretically for a dirty antiferromagnetic superconductor (AFSC), modelling an AFSC as a doped semi-metal with s-wave superconducting pairing and antiferromagnetic (dielectric) interaction between electrons (holes). It is also supposed that the quasiparticles dispersion law possesses the property of nesting. The distribution of the superconducting and magnetic order parameters near the vortex core is calculated. It is shown that the antiferromagnetic order, been suppressed at large distances, is restored around the superconducting flux and the vortex core is in fact insulating and antiferromagnetic, in stark contrast to the normal metal cores of traditional superconductors. Moreover, our model calculations predict that as the temperature decreases the flux region of the superconductivity and antiferromagnetism coexistence increases.
\end{abstract}

PACS numbers:74.20.De, 74.25.Op, 74.25.Ha

\section{INTRODUCTION}

The influence of the antiferromagnetic (AF) correlation in the superconducting state has been attracting much attention recently. In particular, the interrelationship between magnetism and superconductivity (SC) have been actively discussed in connection with the problem of the symmetry type of the order parameter in high- $T_{C}$ systems (see, e.g. ${ }^{1}$ ). It is natural also to ask if the close proximity between antiferromagnetic and superconducting phases could have any macroscopic manifestations. At the macroscopic level, the external magnetic field penetrates type-II superconductors 


\section{V. Garkusha and V. N. Krivoruchko}

via normal state metallic inclusions, or vortices, and can reveal the nature of the system's ground state. So that, by weakening the superconductivity of high- $T_{C}$ due to magnetic field, the interplay between antiferromagnetic and superconducting ordering can be explored. There are predictions that vortices in high- $T_{C}$ cuprates differ from those in conventional superconductors. E.g., Zhang ${ }^{2}$ has developed a unified theory where antiferromagnetism and $d$-wave $\mathrm{SC}$ in high- $T_{C}$ superconductors has been constructed based on $S O(5)$ symmetry. In this theory the AF and $\mathrm{SC}$ order parameters are unified into a five dimensional vector (a superspin). The $S O(5)$ theory predicts the existence of superconducting vortices with antiferromagnetic cores as a spatial class of topological solutions. ${ }^{3}$ Recent magnetic neutron diffraction data, ${ }^{4}$ scanning tunneling microscopy, ${ }^{5}$ muon spin rotation ${ }^{6}$ and nuclear magnetic resonance $^{7}$ measurements on some high- $T_{C}$ SC have revealed that the applied field which imposes the vortex lattice, also induces antiferromagnetic electronic correlations. The new finding is that for optimally doped samples (superconducting material shows no static magnetism in either zero or weak magnetic field) the vortex state has much stronger tendencies towards magnetic order than either the normal or superconducting state. Since magnetism extend beyond the vortex core into the superconducting regions of the material, these result means that antiferromagnetic correlation and superconductivity coexist throughout the bulk of the material, and applied field has only effect of recovering some of the magnetic correlation. ${ }^{3-7}$ One view that this means that the band quasiparticle picture cannot account for the AF order of the vortices and the experimental facts provide good support of the idea of the existence of $S O(5)$ symmetry in high- $T_{C}$ cuprates. (see, e.g., ${ }^{3}$ and references therein).

Recently it was discovered that competition between superconductivity and antiferromagnetism also occur in the new classes of quaternary intermatallic superconductors such as the the borocarbide series $R \mathrm{Ni}_{2} \mathrm{~B}_{2} \mathrm{C}$ and $R N i B C$ (here $R$ - rare-earth ion) (see, e.g., ${ }^{8}$ and references therein). For these new systems, the magnetic energies are dominated by exchange interactions and comparable to the magnetic superconducting condensation energies ensure that the interlay between superocnductivity and antiferromagnetism should be on a comparable energetic footing.

In this report, we study the possibility for antiferromagnetic ordering of the vortex based on the model where the Hamiltonian consists of two components, one corresponding to the (Cooper pair) condensation conditions of equal and opposite momenta and spins and the other to the magnetic interaction condition of parallel spins and finite momentum transfer. A mean-field approach is taken to study the magnetic and superconducting structures of a flux vortex in such AFSC in the so-called "dirty" limit. We 


\section{Vortex core of antiferromagnetic superconductor}

show that the external magnetic field stabilizes the AF order around the vortex core in a region where $\mathrm{SC}$ have been suppressed, i.e., a coexistence phase is induced by the applied field. It was also obtained that the vortex core is, in fact, insulating in stark contrast to the standard metal cores of low-temperature superconductors.

\section{The model}

Selecting the Hamiltonian of an AFSC, we will use the isotropic model of a doped semi-metals with Cooper pairing electrons (holes) within each (two) of the bands and dielectric (electron-hole) pairing between bands. ${ }^{9}$ In the self-consistent field approximation we have

$$
\begin{aligned}
& H=\int d \mathbf{r}\left\{\sum_{\sigma}\left[\psi_{\sigma}^{+} \xi_{\mathbf{1}} \psi_{\sigma}+\varphi_{\sigma}^{+} \xi_{\mathbf{2}} \varphi_{\sigma}\right]\right. \\
& \left.+\left[\Delta_{1 S}(\mathbf{r}) \psi_{\uparrow}^{+} \psi_{\downarrow}^{+}+\boldsymbol{\Delta}_{\mathbf{2} \mathbf{S}}(\mathbf{r}) \varphi_{\uparrow}^{+} \varphi_{\downarrow}^{+}+\mathbf{h . c} .\right]+\boldsymbol{\Delta}_{\mathbf{A}}(\mathbf{r}) \sum_{\sigma}\left[\psi_{\sigma}^{+} \varphi_{\sigma}+\mathbf{h . c} .\right]\right\}(1)
\end{aligned}
$$

Here $\psi_{\sigma}^{+}, \psi_{\sigma}$ and $\varphi_{\sigma}^{+}, \varphi_{\sigma}$ are the creation and annihilation operators of electrons and holes in the first and second bands, respectively, whose dispersion law satisfies the condition of nesting $\xi_{1,2}(\mathbf{p}+\mathbf{Q})=\xi_{\mathbf{p}}$. The fraction of the Fermi surface that is nested, $\nu$, will be treated as a variable. As is known, the Fermi surface nesting causes the transition to spin density wave state. $^{9,10}$ This state can be described by dielectric order parameter, which characterizes the gap in quasiparticle's excitations. For the case of an antiferromagnetic (doubled) cell, we take $\mathbf{Q}=\mathbf{K} / \mathbf{2}$, where $\mathbf{K}$ is the vector of a reciprocal lattice of the crystal. The operator $\xi_{\mathbf{P}}$ is understood to be $\hbar^{2}(\mathbf{p}-\mathbf{e A})^{\mathbf{2}} / \mathbf{2} \mathbf{m}-\mathbf{E}_{\mathbf{F}} ; \Delta_{1(2) S}(\mathbf{r})$ are the band superconducting order parameters; $\Delta_{A}(\mathbf{r})$ is the magnetic order parameter; $E_{F}=\hbar^{2} p_{F}^{2} / 2 m$ stands for the Fermi energy; and $\mathbf{A}$ is the vector potential. As one can see, for the model (1) the SC correlations have the spin-singlet type, while the magnetic ones have the spin-triplet type.

The system of Gor'kov equations for electrons in the first band will be distinguished from the standard system for functions $G_{11}(1,2)=-<$ $T_{\tau} \psi_{\uparrow}(1) \psi_{\uparrow}^{+}(2)>$ and $F_{11}(1,2)=<T_{\tau} \psi_{\downarrow}^{+}(1) \psi_{\uparrow}^{+}(2)>$ by the presence of the additional function $\Im_{21}(1,2)=-<T_{\tau} \varphi_{\downarrow}(1) \psi_{\uparrow}^{+}(2)>$ which describes the $\mathrm{AF}$ correlations of electrons from different bands. (Here we used standard notifications, i.e., $T_{\tau}$ is the $\tau$-ordered operator, $1=\left(\mathbf{r}_{1}, \tau_{\mathbf{1}}\right)$ and so on.) Due to the magnetic interaction between the electrons of different bands, the Green's functions of the second band with a reversed sign for the spin 


\section{V. Garkusha and V. N. Krivoruchko}

appear in set of equations. The complete system of equations contains 12 equations (see Ref.11 for details). In this report the case, when total $S C$ order parameter of the system has the s-wave symmetry, i.e., the case ${ }^{9} \Delta_{1 S}=$ $\Delta_{2 S}=\Delta_{S}$ will be studied. We present here the main equations for such a system in so-called dirty limit. ${ }^{12}$

\section{Quasiclassical equations for dirty AFSC}

There are three characteristic lengths for the system under consideration: the electrons mean free path $\ell$, the SC correlation length $\xi_{S} \sim$ $\hbar v_{F} / \Delta_{S 0}$ and the AF correlation length $\xi_{A} \tilde{\hbar} v_{F} / \Delta_{A 0}$. Here $\Delta_{S 0}$ is the superconducting order parameter if the antiferromagnetic pairing is absent, while $\Delta_{A 0}$ is the antiferromagnetic one when the superconducting pairing is absent. The $\xi_{A}$ can be considered as the characteristic length of electron liquid's magnetic modulation or magnetic stiffness, as well. We will assume that the metal is "dirty" for both orders; i.e., the mean free path $\ell$ and the correlation lengths $\xi_{S}$ and $\xi_{A}$ satisfy the condition $\ell \ll\left(\xi_{S}, \xi_{A}\right)$. Using the standard procedure, one can obtain ${ }^{11}$ the system of equations, that are the generalized Usadel equations for the model (1).

It is convenient ${ }^{11}$ to pass from the band superconducting variables to the total ones: $\Delta_{S}=\Delta_{1 S}+\Delta_{2 S}$, and $F=F_{11}+F_{22}, G_{11}=G_{22}=G$ , $\Im_{21}=\Im_{12}=\Im$. There are tree type of Green's functions: the functions $G \equiv G(\omega, \mathbf{r})$ and $\Im \equiv \Im(\omega, \mathbf{r})$ describe the normal excitations in system of electrons with antiferromagnetic correlations, while the anomalous Green's function $F \equiv F(\omega, \mathbf{r})$ describes the Cooper-pair condensate. These functions are related by the usual normalization condition

$$
G^{2}(\omega, \mathbf{r})+|F(\omega, \mathbf{r})|^{2}+|\Im(\omega, \mathbf{r})|^{2}=1
$$

Magnetic and superconducting properties of the system are described by generalized quasiclassical equations that can be written in the form: ${ }^{11}$

$$
\begin{gathered}
-\frac{1}{2} D \Pi(G \Pi F-F \nabla G)=\frac{\Delta_{S}}{\hbar} G-\omega F \\
D \nabla(G \nabla \Im-\Im \nabla G)=2 \omega \Im-2 \frac{\Delta_{A}}{\hbar} G
\end{gathered}
$$

Where $\boldsymbol{\Pi} \equiv \nabla+2 i \pi \mathbf{A} / \Phi_{0}$ is the gradient-invariant momentum operator, $\Phi_{0}$ stands for the flux quantum, $D=l v_{F} / 3$ is the diffusion coefficient and $\hbar \omega \equiv \hbar \omega_{n}=\pi T(2 n+1)$ is Matsubara frequency ( $T$ is the temperature, $\mathrm{k}_{B}$ $=1$ ). 


\section{Vortex core of antiferromagnetic superconductor}

These equations should be supplemented by formulas for the order parameters and relation between supercurrent and vector potential. The selfconsistency conditions for the order parameters $\Delta_{S}$ and $\Delta_{A}$ are

$$
\begin{aligned}
& \Delta_{S} \ln \left(T / T_{C 0}\right)+2 \pi T \sum_{\omega>0}\left(\Delta_{S} / \hbar \omega-F\right)=0 \\
& \Delta_{A} \ln \left(T / T_{N 0}\right)+2 \pi T \sum_{\omega>0}\left(\Delta_{A} / \hbar \omega-\Im\right)=0
\end{aligned}
$$

Here $T_{C 0}$ is the superconducting transition temperature when antiferromagnetic pairing is absent, while $T_{N 0}$ is the antiferromagnetic transition temperature when these is no superconducting pairing. The self-consistency condition for the vector potential has the usual form (London electrodynamics):

$$
\nabla \times(\nabla \times \mathbf{A})=\frac{4 \pi}{c} j_{S}=i \frac{4 \pi}{c} e N(0) D T \sum_{\omega>0}(F \Pi F+\text { h.c. })
$$

where $N(0)$ is the density of states on the Fermi surface. If an antiferromagnetic pairing is vanished the Eqs. (2), (3), (5) and (7) restore those for a nonmagnetic superconductor.

Assuming the two order parameters to be spatially homogeneous, the model (1) leads to four possible states, each with a specific solutions $\left(\Delta_{S}, \Delta_{A}\right)$. Namely: (i) the normal state $(0,0)$; (ii) the BCS superconducting state $\left(\Delta_{S}, 0\right)$; (iii) a purely antiferromagnetic state $\left(0, \Delta_{A}\right)$; and (iv) a coexistence phase where both $\Delta_{S}$ and $\Delta_{A}$ are non-zero. The phase with the lowest free energy is stable. The phase diagram for the system can be calculated in terms of the relative interaction strengths of the two orders (the relationship $T_{C 0} / T_{N 0}$ ) and the fraction of the Fermi surface that is nested (the results will be published elsewhere. ${ }^{13}$ ) We will not analyze here all the phases but only the BCS one when in the sample bulk the antiferromagnetic order is suppressed. That means that the boundary conditions for Eqs. (2)-(4)

$$
G_{0}=\hbar \omega / \sqrt{(\hbar \omega)^{2}+\Delta_{S 0}^{2}}, F_{0}=\Delta_{S 0} / \sqrt{(\hbar \omega)^{2}+\Delta_{S 0}^{2}}, \Im_{0}=0
$$

match the solutions to the Green's function at large distances. Here $\Delta_{S 0}$ is the superconducting order parameter if antiferromagnetic pairing is absent.

\section{Basic equations in the vortex geometry}

To study the vortex it is convenient to work in the gauge in which $F$ and $\Delta_{S}$, and $\Im$ and $\Delta_{A}$ are real. The coordinate dependence of all functions 


\section{V. Garkusha and V. N. Krivoruchko}

is then only through the distance $r$ from the axis of the vortex and we may define $\varphi \equiv \varphi\left(\omega_{n}, r\right)=\varphi_{n}$ and $\theta \equiv \theta\left(\omega_{n}, r\right)=\theta_{n}$ by such a way that the Eq.(2) is fulfilled:

$$
G(\omega, r)=\cos \varphi \cos \theta, F(\omega, r)=\sin \varphi \cos \theta, \Im(\omega, r)=\sin \theta
$$

Due to the cylinder symmetry of a vortex the lines of $\mathbf{A}$ are circles around it and the relations $\nabla \mathbf{A}=0, \mathbf{A} \nabla \varphi=0$ and $\mathbf{A} \nabla \theta=0$ took place. By making use these relations, the equations of motions for $\varphi$ and $\theta$ can be obtained from Eqs. (3) and (4) in the form:

$$
\begin{gathered}
\xi^{2}\left\{\cos \theta\left(\nabla^{2} \varphi\right)-2 \sin \theta(\nabla \varphi)(\nabla \theta)\right\}= \\
\frac{1}{2} \xi^{2}\left(\frac{2 \pi \mathbf{A}}{\Phi_{0}}\right)^{2} \sin 2 \varphi \cos \theta+\frac{\Delta_{S}(r)}{\Delta_{S 0}} \cos \varphi-\frac{\hbar \omega}{\Delta_{S 0}} \sin \varphi \\
\xi^{2}\left\{\cos \varphi\left(\nabla^{2} \theta\right)+\sin \theta\left[\sin \varphi \cos \theta\left(\nabla^{2} \varphi\right)+\cos \varphi \cos \theta(\nabla \varphi)^{2}-2 \sin \varphi \sin \theta(\nabla \varphi)(\nabla \theta)\right]\right\} \\
=\frac{\hbar \omega}{\Delta_{S 0}} \sin \theta-\frac{\Delta_{A}(r)}{\Delta_{S 0}} \cos \varphi \cos \theta
\end{gathered}
$$

where $\xi=\left(D / 2 \Delta_{S 0}\right)^{1 / 2}$ stands for the SC correlation length. The equation for the vector potentials now is took the form

$$
\nabla \times(\nabla \times \mathbf{A})=-\frac{4 \pi}{c} Q \mathbf{A},
$$

where the diamagnetic response function $Q$ reads

$$
Q=4 \pi e^{2} N(0) D T / \hbar c \times 2 \pi \sum_{\omega>0} \sin ^{2} \varphi \cos ^{2} \theta
$$

At large distances from the flux axis $(r \rightarrow \infty)$, in accordance with the boundary conditions (8) we have $\varphi \rightarrow \varphi_{\infty}=\operatorname{arctg}\left(\Delta_{S 0} / \omega\right)$ and $\theta \rightarrow \theta_{\infty}=0$. Then $Q \rightarrow Q_{0}=c / 4 \pi \lambda^{2}$ where $\lambda$ is the London penetration depth is given by the usual dirty limit formula ${ }^{14}$

$$
\lambda^{-2}=\frac{8 \pi^{2}}{\hbar c^{2}} e^{2} N(0) D \tanh \frac{\Delta_{S 0}}{2 T}
$$

In the limit of extreme type-II SC the solution to Eqs. (10), (11) and (12) may be developed by a series of powers of Ginzburg-Landau parameter $1 / \kappa^{2}$ $(\kappa=\lambda / \xi)$. We will calculate the results up to $O\left(1 / \kappa^{4}\right)$ terms. Note, that for $\kappa \gg 1$ and $\xi \leq r$ the expression for vector potential is dominated by the largest term, which, as follows from Eq. (12), is

$$
A(r)=-\frac{\hbar c}{2 e \lambda} K_{1}(r / \lambda) \text {. }
$$




\section{Vortex core of antiferromagnetic superconductor}

Here $K_{1}(x)$ is the imaginary Bessel function of first order. Note that if $\Delta_{A} \equiv 0, \theta_{n} \equiv 0$ our formulas restore those considered by Watts-Tobin and Waterworth. ${ }^{15}$

For the purposes of computation, it is convenient to choose $R=r / \xi$ as the independent variable and to obtain

$$
\begin{aligned}
& \frac{d^{2} \varphi_{n}}{d R^{2}}+\frac{1}{R} \frac{d \varphi_{n}}{d R}=\frac{1}{2} \kappa^{-2} \sin 2 \varphi_{\infty} K_{1}^{2}(R / \kappa)-\frac{\Delta_{S}(R)}{\Delta_{S 0}} \frac{\cos \varphi_{n}}{\cos \theta_{n}}+\frac{\hbar \omega_{n}}{\Delta_{S 0}} \frac{\sin \varphi_{n}}{\cos \theta_{n}} \\
& \frac{d^{2} \theta_{n}}{d R^{2}}+\frac{1}{R} \frac{d \theta_{n}}{d R}=\left\{\frac{\Delta_{S}(R)}{\Delta_{S 0}} \sin \varphi_{n}+\frac{\hbar \omega_{n}}{\Delta_{S 0}} \cos \varphi_{n}\right\} \sin \theta_{n}-\frac{\Delta_{A}(R)}{\Delta_{S 0}} \cos \theta_{n}
\end{aligned}
$$

Here in the terms of $\propto \kappa^{-2}$, the zero-order values of $\mathbf{A}(r), \varphi(r)$ and $\theta(r)$ are used. Accordingly, the self-consistency conditions now are determined by

$$
\begin{gathered}
\Delta_{S}(R)=2 \pi T \sum_{n \geq 0} \sin \varphi_{n} \cos \theta_{n}\left\{\ln \frac{T}{T_{C 0}}+2 \pi T \sum_{n \geq 0} 1 / \hbar \omega_{n}\right\}^{-1} \\
\Delta_{A}(R)=2 \pi T \sum_{n \geq 0} \sin \theta_{n}\left\{\ln \frac{T}{T_{N 0}}+2 \pi T \sum_{n \geq 0} 1 / \hbar \omega_{n}\right\}^{-1}
\end{gathered}
$$

and $\Delta_{S 0}$ is determined by the relation (18) with $\cos \theta_{n}=1$.

We solved these set of equations numerically by an iteration procedure. The results of our calculations are presented in Figs. 1-3, where the SC and AF order parameters near the vortex core, $\Delta_{S}(R)$ and $\Delta_{A}(R)$, respectively, are shown for different values of the $T_{C 0} / T_{N 0}$ ratio, for a given fraction of the Fermi surface that is nested and at different temperatures. The solid line represents a coordinate dependence of the superconducting order parameter $\Delta_{S}(R)$; the dashed line depicts the antiferromagnetic order parameter $\Delta_{A}(R)$ behavior. As it is seen in the figures, the size of the "antiferromagnetic" vortex core is on the order of the superconducting coherence length. I.e., the model predicts that the vortex core is in fact insulating and antiferromagnetic. Moreover, as $T / T_{C 0}$ decreases the region near the flux core where superconductivity and antiferromagnetism to coexist increases.

\section{Summary}

We investigated the structure of an isolated single vortex for a dirty metal with competing superconducting (a spin singlet pairing) and antiferromagnetic (a spin triplet pairing) interactions. We shown that the external magnetic field stabilized the antiferromagnetic order around the superconducting core, in the region where superconductivity has been suppressed. It is obtained that the vortex core is insulating and antiferromagnetic, in stark 


\section{V. Garkusha and V. N. Krivoruchko}

contrast to the standard normal metal cores of traditional superconductors. The main our nontrivial result is that as $T / T_{C 0}$ decreases the region where superconductivity and antiferromagnetism to coexist increases.

In recent years, one can see an explosion in the field of vortex dynamics, especially in the materials of high- $T_{C} \mathrm{SC}$. As is well known, inertial mass of a fluxion play an important role in vortex dynamics. There are various mechanisms contributing to the flux inertial mass, and the important one is due to variation of local electronic density of state in Abrikosov vortices. The metallic core with states very close to the Fermi energy predicted by Caroly et. $a l .{ }^{16}$ is the standard picture for discussion of inertial mass of the vortex. However, the static, long-ranged, magnetism associated with the vortex core may have nontrivial influence upon transport properties of magnetic superconductors in an applied magnetic field and should be taken into account for systems with competition between superconductivity and antiferromagnetism.

The authors are grateful to V. Chabanenko and N. Hayashi for stimulating discussions.

\section{REFERENCES}

[1] A. P. Kampf, Phys. Rep., 249 , 219 (1994).

[2] S.-C. Zhang, Science 275, 1089 (1997).

[3] D. P. Arovas, A. J. Berlinsky, C. Kallin, and S.-C. Zhang, Phys. Rev. Lett. 79, 2871 (1997); S. Alama, A. J. Berlinsky, L. Bronsard and T. Giorgi, Phys. Rev. B 60, 6901 (1999); H. Bruus, K. A. Eriksen, M. Hallundbæk, and P. Hederård, B 59, 4349 (1999).

[4] S. Katano, M. Sato, K. Yamada, et al., Phys. Rev. B 62, R14677 (2000); B. Lake, G. Aeppli, K. N. Clausen, et al., Science 291, 832 (2001); B. Lake, H. M. Ronnow, N. B. Christensen, et al., Nature 415, 299 (2002).

[5] Ch. Renner, B. Revaz, K. Kadovski, I. Maggio-Aprile, and Ø. Fischer, Phys. Rev. Lett. 80, 3606 (1998).

[6] R. Kadono, W. Higemoto, A. Koda, et al. Phys. Rev. B 69, 104523 (2004).

[7] V. F. Mitrović, E. E. Sigmund, M. Eschring, et al. Nature 413, 501 (2001).

[8] J. W. Lynn, S. Skanthakumar, Q. Huang, et al. Phys. Rev. B 55, 6584 (1997).

[9] Yu. V. Kopaev, Sov. Phys. Usp. 32, 1033 (1989) [Usp. Fiz. Nauk 159, 567 (1989)].

[10] G. Güner. Rev. Mod. Phys. 66, 1 (1994).

[11] V. N. Krivoruchko, JETP 84, 300 (1997) [Zh. Ėksp. Teor. Fiz. 111, 547(1997)].

[12] K.-D. Usadel, Phys.Rev.Lett. 25, 560 (1970).

[13] V. V. Garkysha and V. N. Krivoruchko, to be published.

[14] P. G. de Gennes, Superconductivity of Metals and Alloys (Benjamin, NY, 1966).

[15] R. J. Watts-Tobin and G. M. Waterworth, Z.Phys. 261, 249 (1973).

[16] C.Caroly, P.G. de Gennes, J. Matricon. J. Phys. Lett. (Fr.) 9, 307 (1964). 


\section{Vortex core of antiferromagnetic superconductor}

Fig. 1 Radial coordinate dependence of the vortex superconducting order parameter $\Delta_{S}(R)$ (solid lines) and antiferromagnetic order parameter $\Delta_{A}(R)$ (dashed lines) for $\kappa=10$ and different values of $T / T_{C 0}=0.05,0.5$, and 0.8. $T_{C 0}=T_{N 0} ; \nu \leq 1$. Fig. 2 Same as in Fig. 1 for $T_{C 0}=0.5 T_{N 0}$, $\nu \leq 0.5$. Fig. 3 Same as in Fig. 1 for $T_{C 0}=2 T_{N 0}, \nu \leq 1$. 


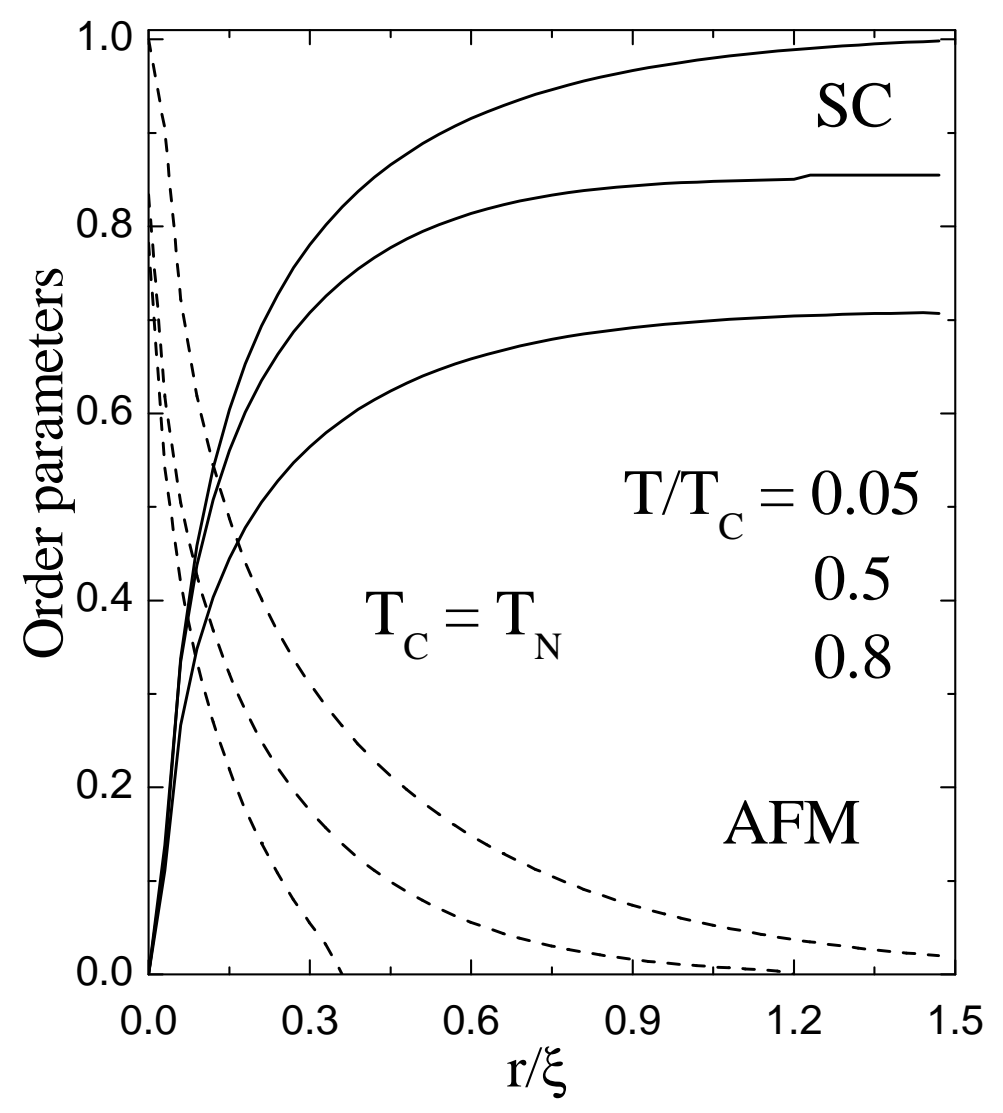




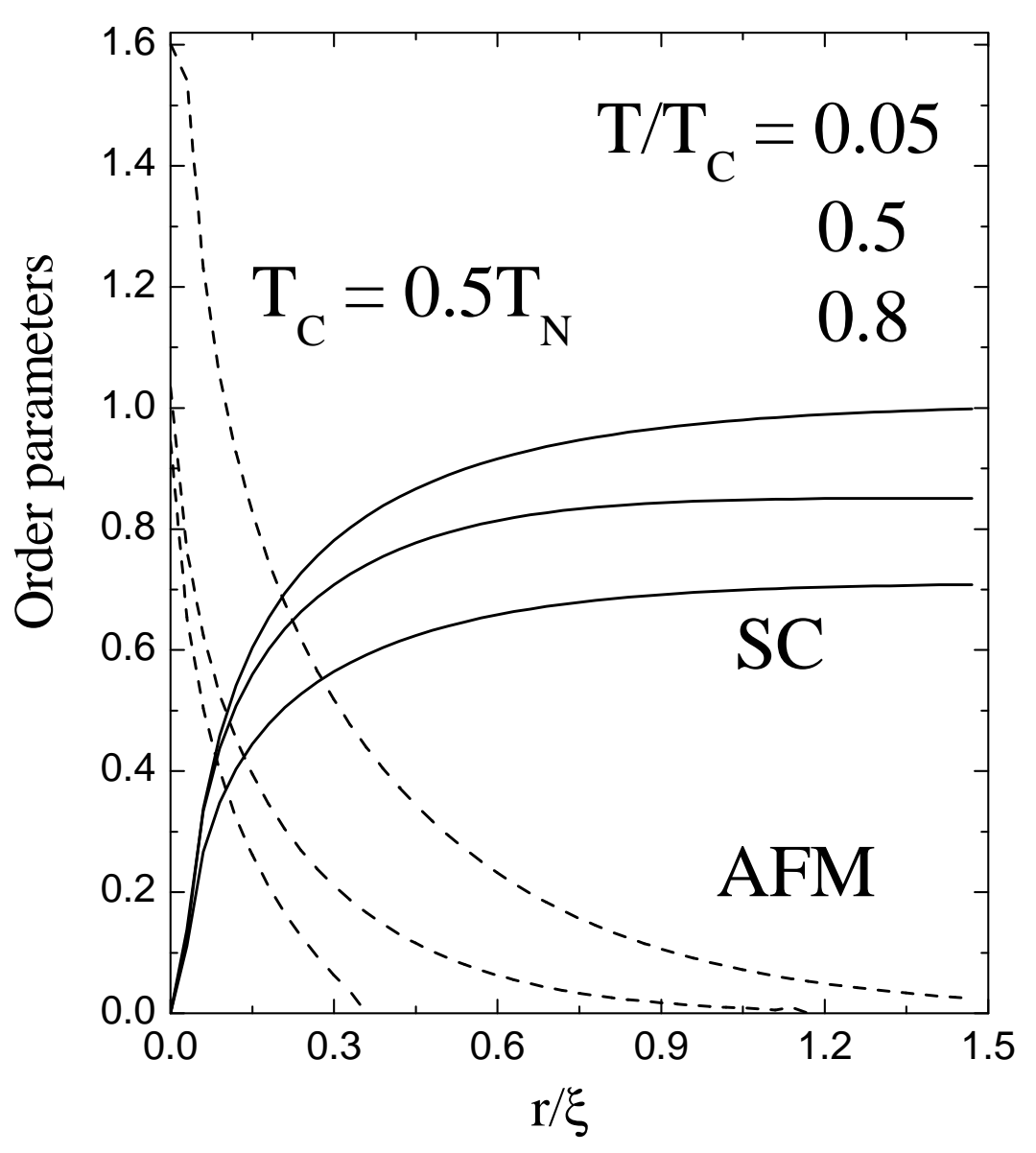




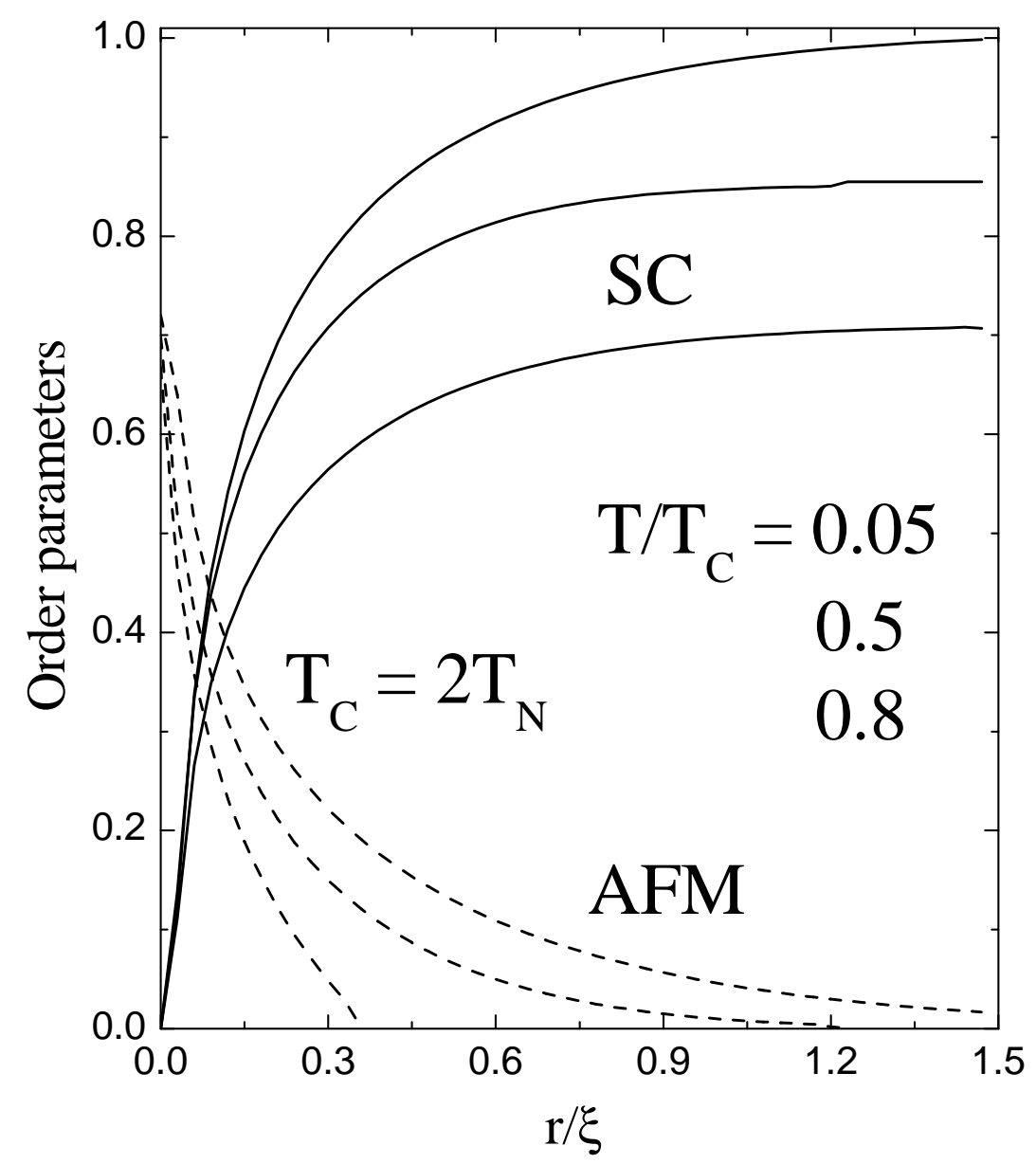

